\title{
Breathing Patterns of Healthy Human Response to Different Levels of Physical Activity
}

\author{
Endalew Getnet Tsega*, V.K. Katiyar, Pratibha Gupta \\ Department of Mathematics, Indian Institute of Technology Roorkee, Roorkee, India \\ *Corresponding author: endalebdumath2016@gmail.com \\ Received April 14, 2019; Revised May 22, 2019; Accepted June 09, 2019
}

\begin{abstract}
Many people nowadays do physical activities to improve long-term health, strengthen muscles and bones and get emotional and mental benefits. During physical activity, rate and depth of breathing increase because working muscles need extra oxygen in order to produce more energy. The purpose of this study is to investigate the breathing pattern of healthy human in response to different levels of physical activity measured by distance and duration. Spirometric measurement of breathing parameters of five male participants at rest (sitting and standing) and response to different levels of physical activity (walking, jogging and running) was performed. Using the spirometry data, mathematical models were developed to describe the resulting breathing patterns. The data and the model were used for the analysis of breathing patterns. There was small variation of corresponding breathing parameters for a subject during sitting and standing at rest. Increasing the level of physical activity raised the respiratory characteristic such as tidal volume, breathing frequency and flow rate. The average and peak inspiratory and expiratory flow rates were obtained for the five breathing conditions. The peak inspiratory air flow rate in response to running was 3.4 times that of walking. It was 3.7 times for expiration. Breathing patterns of any two individuals are different. The human breathing is highly affected by the level of physical activity we are doing. The study can help to create awareness regarding breathing pattern related to physical activity and to choose appropriate physical activity.
\end{abstract}

Keywords: physical activity, healthy, respiratory response, breathing parameters, sinusoidal curve

Cite This Article: Endalew Getnet Tsega, V.K. Katiyar and Pratibha Gupta, "Breathing Patterns of Healthy Human Response to Different Levels of Physical Activity." Journal of Biomedical Engineering and Technology, vol. 7, no. 1 (2019): 1-4. doi: 10.12691/jbet-7-1-1.

\section{Introduction}

Physical activity is any bodily movement produced by the musculoskeletal system resulting in energy expenditure [1]. It includes activities undertaken in day-to-day living, health improvement, physical fitness, exercises and recreational sports. During physical activity, the working muscles require more energy and so extra oxygen is needed to release this energy from glucose [2]. As the level of activity is increased, breathing rate increases to bring more oxygen from air. This alters the respiratory system physiology [3]. It is important to understand and quantify the respiratory changes in response to a given level of physical activity.

Numerous studies have been carried out to investigate the work of breathing in response to physical activity. Guenette et al. [4] investigated respiratory response to exercise in endurance-trained men and women. They considered different breathing parameters including tidal volume, breathing rate, minute ventilation, and duration of inspiration and expiration. Mihailova and Kaminski [5] examined the relation between body mass index, amount of physical activity, aerobic fitness and breathing parameters in students. Johnson [6] summarized the cardiorespiratory responses to different levels of exercise including walking, jogging and running for a normal young male. Serna et al. [7] developed a computational model of respiratory system in dynamic response to exercise. Variables related to breathing pattern and gas exchange were included in the model. Di Paco et al. [8] used ventilatory parameters to evaluate professional soccer players' performance. Relation between minute ventilation and oxygen uptake at peak treadmill exercises was constructed for the study. Treadmill has been commonly used to investigate cardiorespiratory responses to physical activities [9,10,11]. Wu et al. [12] used a finite state model to quantify respiratory motion characteristics. It is important to understand and quantify the breathing parameters responses to a given level of physical activity for analysis of airflow dynamics in the human respiratory tract.

\section{Anthropometric and Respiratory Data}

Five healthy male subjects who finished all the designed physical activities completely were taken in the 
study. Breathing parameters of the subjects at rest (sitting and standing) were measured using microQuark PC-based spirometer from COSMED with SVC test by a Laboratory at Patanjali Research Foundation, India. Measurements were taken from mouth ventilation alone by closing nostrils with nose clips. A JKEXER treadmill with no incline was arranged in the same room and close to the spirometer. The subjects performed walking, jogging and running on the treadmill with a speed of $4 \mathrm{~km} / \mathrm{hr}, 7 \mathrm{~km} / \mathrm{hr}$ and $10 \mathrm{~km} / \mathrm{hr}$ respectively with in a fixed distance of $500 \mathrm{~m}$. The time gap between the physical activities was 20 minutes for all participants. Breathing parameters were measured immediately at the end of each activity. Three trial SVC tests in three different days were taken for the participants in response to sitting at rest, standing at rest, walking, jogging and running. For each physical activity, the trial having median reading of tidal volumes was selected as the representation of the respiratory data for this study.

Anthropometric characteristics of the subjects are shown in Table 1. Table 2 represents the statistical data of breathing parameters measured response to different levels of physical activity. In Table 2, $V_{T}$ is tidal volume, $f$ is respiratory frequency, $T_{i}$ is inspiration time and $T_{e}$ is expiration time. The total respiratory cycle time $T_{\text {tot }}$ can be calculated as $T_{\text {tot }}=T_{i}+T_{e}$.
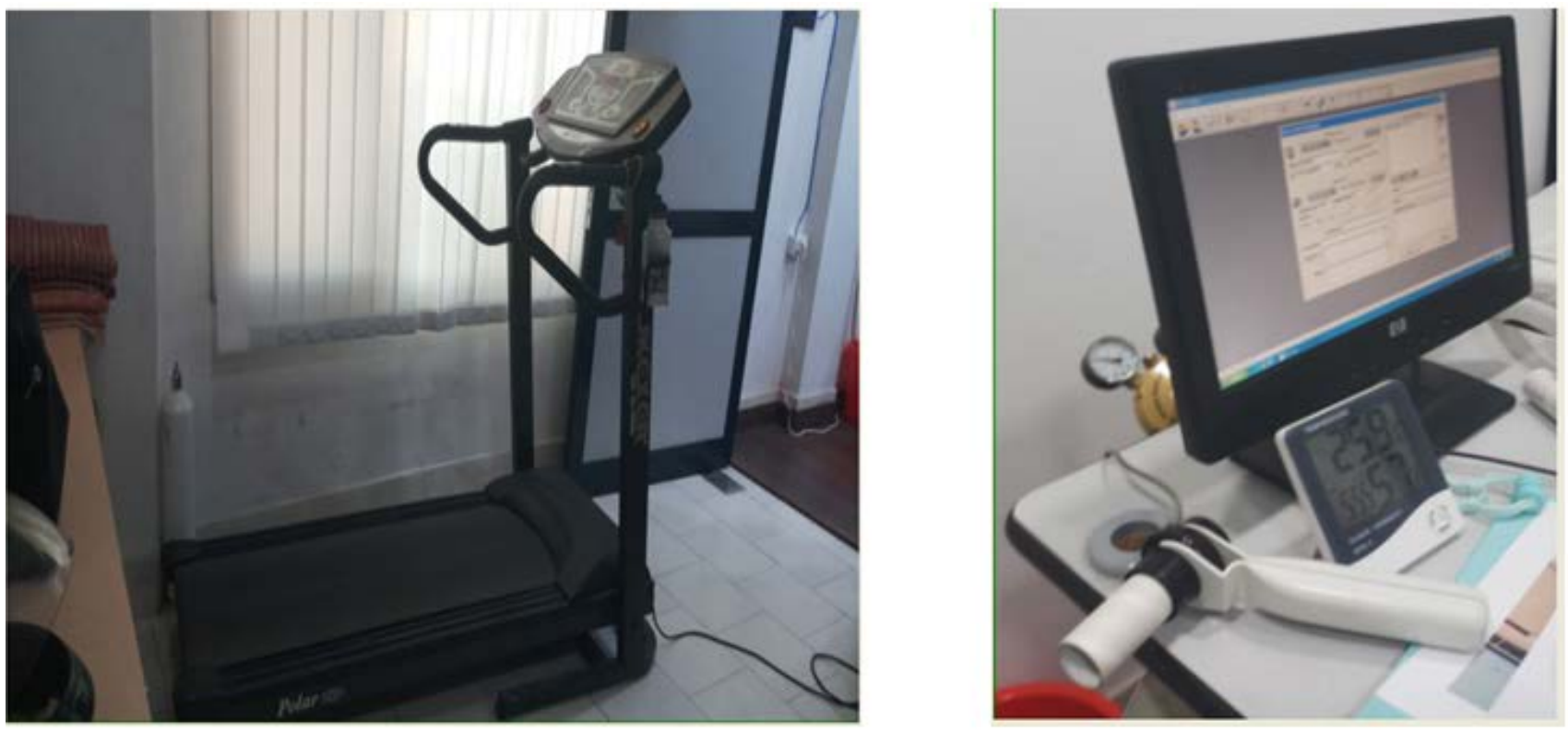

Figure 1. Photograph of treadmill and spirometer used in the study

Table 1. Anthropometric characteristics of the subjects

\begin{tabular}{|l|r|}
\hline Variables & Mean \pm SD \\
\hline Number & 5 \\
\hline Age(year) & $29.6 \pm 6.62$ \\
\hline Height $(\mathrm{cm})$ & $166.00 \pm 6.23$ \\
\hline Weight(kg) & $63.96 \pm 10.41$ \\
\hline BMI $\left(\mathrm{kg} / \mathrm{m}^{2}\right)$ & $23.22 \pm 3.72$ \\
\hline
\end{tabular}

Table 2. Over all mean values $( \pm \mathrm{SD})$ obtained from spirometric measurement of five subjects' breathing parameters response to different levels of physical activities in $500 \mathrm{~m}$

\begin{tabular}{|c|c|c|c|c|c|}
\hline Parameter & Sitting at rest & Standing at rest & Walking $(4 \mathrm{~km} / \mathrm{hr})$ & Jogging (7km/hr) & Running $(10 \mathrm{~km} / \mathrm{hr})$ \\
\hline$V_{T}(\mathrm{~L})$ & $0.48 \pm 0.02$ & $0.54 \pm 0.07$ & $0.98 \pm 0.07$ & $1.54 \pm 0.14$ & $2.01 \pm 0.13$ \\
\hline f(breaths/min) & $12.71 \pm 0.48$ & $13.54 \pm 1.11$ & $20.12 \pm 2.46$ & $30.44 \pm 1.11$ & $34.50 \pm 1.00$ \\
\hline$T_{i}(\mathrm{~s})$ & $1.86 \pm 0.06$ & $1.80 \pm 0.17$ & $1.38 \pm 0.19$ & $0.94 \pm 0.07$ & $0.84 \pm 0.06$ \\
\hline$T_{e}(\mathrm{~s})$ & $2.87 \pm 0.12$ & $2.65 \pm 0.18$ & $1.61 \pm 0.22$ & $1.05 \pm 0.11$ & $0.90 \pm 0.10$ \\
\hline
\end{tabular}




\section{Sinusoidal Models of the Breathing Patterns}

The flow-time curves displayed on the PC screen for the breathing conditions were assumed to be sinusoidal $[13,14]$. Suppose $Q_{i}$ is an inspiratory flow rate at the mouth inlet and $V_{T}$ is the corresponding tidal volume for flow with

$$
Q_{i}(t)=Q_{P I F} \sin \left(\frac{\pi t}{T_{i}}\right)
$$

where $Q_{P I F}$ is the peak inspiratory flow rate and $0 \leq t \leq T_{i}$ [6]. Since the accumulated inspiratory airflow equals to the tidal volume, we have

$$
V_{T}=\int_{0}^{T_{i}} Q_{P I F} \sin \left(\frac{\pi t}{T_{i}}\right) d t
$$

Evaluating the integral in Eq. (2) and solving for $Q_{P I F}$, we get $Q_{P I F}=\frac{\pi V_{T}}{2 T_{i}}$. Hence the inspiratory flow rate during inspiration time is given by

$$
Q_{i}(t)=\frac{\pi V_{T}}{2 T_{i}} \sin \left(\frac{\pi t}{T_{i}}\right), 0 \leq t \leq T_{i}
$$

Similarly, assume $Q_{e}$ is an expiratory flow rate and

$$
Q_{e}(t)=Q_{P E F} \sin \left(\pi+\frac{\pi\left(t-T_{i}\right.}{T_{e}}\right),
$$

where $Q_{P E F}$ is the peak expiratory flow rate and $T_{i}<t \leq T_{i}+T_{e}$. Applying the same procedure as the inspiration, we get

$$
Q_{e}(t)=\frac{\pi V_{T}}{2 T e} \sin \left(\pi+\frac{\pi\left(t-T_{i}\right.}{T_{e}}\right),
$$

where $T_{i}<t \leq T_{i}+T_{e}$. If $Q(t)$ is the respiratory flow with $0 \leq t \leq T$ where $T=T_{i}+T_{e}$ is the respiratory cycle time, using Eq. (3) and Eq. (5), we get

$$
Q(t)= \begin{cases}\frac{\pi V_{T}}{2 T_{i}} \sin \left(\frac{\pi t}{T_{i}}\right), & 0 \leq t \leq T_{i} \\ \frac{\pi V_{T}}{2 T_{e}} \sin \left(\pi+\frac{\pi}{T_{e}}\left(t-T_{i}\right)\right), & T_{i} \leq t \leq T_{i}+T_{e}\end{cases}
$$

Figure 2 shows the sinusoidal flow-time curves for complete respiratory cycle time response to sitting at rest, standing at rest, walking, jogging and running using Eq.(6). In Figure 2, flow rates during expiration are represented by negatives.

\section{Results and Discussion}

From the data, it is observed that the inspiration time is less than the expiration time in each breathing condition.
If the level of the physical activity is increased, the respiratory time decrease and the inspiratory to expiratory time ratio approaches to 1 which agrees with the finding of Anderson et al. [15]. The average inspiratory flow rate ( $\left.V_{T} / T_{i}\right)$ and average expiratory flow rate $\left(V_{T} / T_{e}\right)$ at the mouth inlet can be obtained by simple calculation using the data from Table 2 for each breathing condition. The average inspiratory flow rates in response to sitting at rest, standing at rest, walking, jogging and running were $0.26,0.32,0.79,1.88$ and $2.51 \mathrm{~L} / \mathrm{s}$ respectively. The corresponding values for expiration were $0.17,0.22,0.71$, 1.72 and $2.24 \mathrm{~L} / \mathrm{s}$.

Using the mathematical models, the peak inspiratory flow rates response to sitting at rest, standing at rest, walking, jogging and running were found to be $0.41,0.47$, 1.12, 2.57 and $3.76 \mathrm{~L} / \mathrm{s}$ respectively. The corresponding peak expiratory flow rates were $0.26,0.32,0.96,2.30$ and $3.51 \mathrm{~L} / \mathrm{s}$. It was observed that there was small difference between the breathing patterns during sitting and standing at rest [16]. Increasing the level of physical activity significantly changes respiratory frequency, tidal volume and the respiratory cycle time of healthy human $[17,18]$. The peak inspiratory air flow rate in response to running was 3.4 times (increased by $70 \%$ ) that of walking. It was 3.7times (increased by 73\%) for expiration.

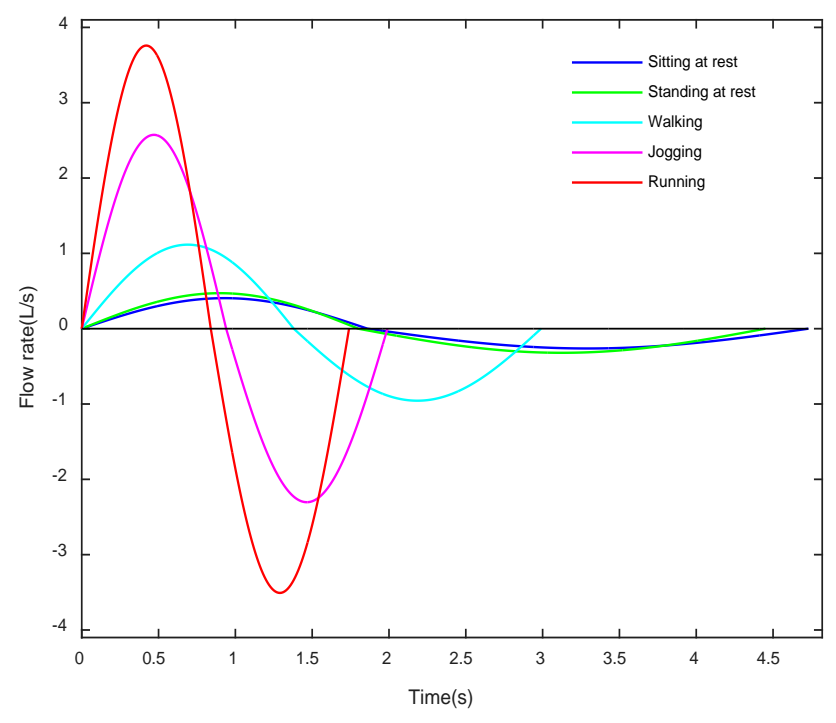

Figure 2. Flow-time curves for the different breathing conditions

\section{Conclusions}

In this study, spirometry data is used to describe breathing patterns of health human response to different levels of physical activity and to develop mathematical models for further analysis of the breathing patterns. Breathing patterns of any two individuals are different when they are at rest or engaged in physical activities. As the level of activity increased, the respiratory characteristic such as tidal volume, breathing frequency and flow rate raised. The changes are described quantitatively in the study. The study can help to create awareness regarding breathing pattern related to physical activity and to choose appropriate physical activity. It may provide a good insight to obtain input data for performing 
numerical simulation of airflow dynamics in human airways under different breathing conditions.

\section{Conflict of Interest Statement}

The authors declare that they have no conflicts of interest.

\section{Acknowledgments}

The authors thank Dr. Shirley Telles, Director of Research, Patanjali Research Foundation, Haridwar, India, for her kind permission to use necessary equipments and support in collecting spirometry data. We also acknowledge Deepak Kumar Pal and Kumar Gandharva, staff members in the Foundation, for providing precious support during the data collection. We also thank all the subjects for their participation in the study.

\section{References}

[1] Caspersen, C.I., Powell, K.E., Christenson, G.M., 1985. Physical activity, exercise and physical fitness: definitions and distinctions for health-related research. Public health Reports 100, 126-131.

[2] Baker, J. S., McCormick, M.C., Robergs, R. A., 2010. Interaction among skeletal muscle metabolic energy systems during intense exercise. Journal of Nutrition and Metabolism.

[3] Katch, V. L., McArdle, W. D., Katch, F. I., 2011. Essentials of Exercise Physiology. Fourth edition, Philadelphia, Pennsylvania, pp. 263-300.

[4] Guenette, J. A., Witt, J. D., McKenzie, D. C., Road, J. D., Sheel A.W., 2007. Respiratory mechanics during exercise in endurancetrained men and women. Journal of Physiology 581, 1309-1322.

[5] Mihailova. A., Kaminska, I., 2016. Lung volumes related to physical activity, physical fitness, aerobic capacity and body mass index in students. SHS Web of Conferences 30, 00017.
[6] Johnson, A.T., 2007. Biomechanics and Exercises Physiology: Quantitative Modeling. Second edition, CRC press, Boca Raton, FL, USA.

[7] Serna, L.Y., Mañanas, M.A., Hernández, A.M., Rabinovich, R.A., 2018. An improved dynamic model for the respiratory response to exercise. Frontiers in Physiology, 9: 69.

[8] Di Paco, A., Catapano, G. A., Vagheggini G., Mazzoleni, S., Micheli L. M. Ambrosino, N., 2014. Ventilatory response to exercise of elite soccer players. Multidisciplinary Respiratory Medicine, 9:20.

[9] Mcllroy, M. B., 1963. The respiratory response to exercise. Pediatrics, 32:4.

[10] de Castro, R. R., Lima, S. P., Sales, A. R. K., da Nóbrega, A. C.L., 2017. Minute-ventilation variability during cardiopulmonary exercise test is higher in sedentary men than in athletes. Arq Bras Cardiol 109(3): 185-190.

[11] Guenette, J. A., Chin, R. C, Cory J.M., Webb K. A., O'Donnell, D.E., 2013. Inspiratory capacity during Exercise: measurement, analysis, and interpretation. Pulmonary Medicine.

[12] Wu, H., Sharp, G.C, Salzberg, B., Kaeli, D, Shirato, H., Jiang S.B., 2004. A finite state model for respiratory motion analysis in image guided radiation therapy. Physics in Medical and Biology 49, 5357-72.

[13] Scheinherr, A. Bailly, L. Boiron, O. Lagier, A. Legou, T. Pichelin. M. Caillibotte, G. Giovanni, A., 2015. Realistic glottal motion and airflow rate during human breathing. Medical Engineering \& Physics 37, 829-839.

[14] Honda, K., Maeda, S. 2008. Glottal-opening and airflow pattern during production of voiceless fricatives: a new non-invasive instrumentation. The Journal of the Acoustical Society of America 123, p.3738.

[15] Anderson, N.J., Cassidy, P.E., Janssen, L.L., Dengel, D.R., 2006 Peak inspiratory flows of adults exercising at light, moderate and heavy work Loads. Journal of the International Society for Respiratory Protection 23, 53-63.

[16] Price, K., Schartz, P., Watson, A.H. 201. The effect of standing and sitting postures on breathing in brass players. Springer Plus 3 : 210

[17] Neder, J.A, Dal Corso, S, Malaguti, C., Reis, S., De Fuccio, M.B., Schmidt, H., Fuld, J.P., Nery, L.E., 2003. The pattern and timing of breathing during incremental exercise: a normative study. European Respiratory Journal 21, 530-538.

[18] Paek, D., McCool, F. D., 1992. Breathing patterns during varied activities. Journal Applied Physiology 73, 887-893.

C The Author(s) 2019. This article is an open access article distributed under the terms and conditions of the Creative Commons Attribution (CC BY) license (http://creativecommons.org/licenses/by/4.0/). 